\title{
A new explicit iterative algorithm for solving a class of variational inequalities over the common fixed points set of a finite family of nonexpansive mappings
}

Cuijie Zhang* and Caiping Yang

\section{"Correspondence:}

zhang_cui_jie@126.com

College of Science, Civil Aviation

University of China, Tianjin, 300300,

P.R. China

\begin{abstract}
In this paper, we introduce a new explicit iterative algorithm for finding a solution for a class of variational inequalities over the common fixed points set of a finite family of nonexpansive mappings in Hilbert spaces. Under suitable assumptions, we prove that the sequence generated by the iterative algorithm converges strongly to the unique solution of the variational inequality. Our result improves and extends the corresponding results announced by many others. At the end of the paper, we extend our result to the more broad family of $\lambda$-strictly pseudo-contractive mappings.
\end{abstract}

Keywords: nonexpansive mapping; strong convergence; variational inequalities; common fixed points

\section{Introduction}

Let $H$ be a real Hilbert space with inner product $\langle\cdot, \cdot\rangle$ and norm $\|\cdot\|$. Throughout this paper, we always assume that $T$ is a nonexpansive operator on $H$. The fixed point set of $T$ is denoted by $\operatorname{Fix}(T)$, i.e., $\operatorname{Fix}(T)=\{x \in H: T x=x\}$. The typical problem is to minimize a quadratic function on a real Hilbert space $H$ :

$$
\min _{x \in C} \frac{1}{2}\langle A x, x\rangle-\langle x, u\rangle
$$

where $C$ is a nonempty closed convex subset of $H, u$ is a given point in $H$ and $A$ is a strongly positive bounded linear operator on $H$.

In 2003, Xu [1] introduced the following iterative scheme:

$$
x_{n+1}=\alpha_{n} u+\left(I-\alpha_{n} A\right) T x_{n},
$$

where $u$ is some point of $H$ and $\left\{\alpha_{n}\right\}$ is a sequence in $(0,1)$. He proved that the sequence $\left\{x_{n}\right\}$ converges strongly to the unique solution of the minimization problem (1.1) with $C=$ $\operatorname{Fix}(T)$.

In 2006, Marino and Xu [2] considered the viscosity method on the iterative scheme (1.2), and they gave the following general iterative method:

$$
x_{n+1}=\alpha_{n} \gamma f\left(x_{n}\right)+\left(I-\alpha_{n} A\right) T x_{n},
$$

○2014 Zhang and Yang; licensee Springer. This is an Open Access article distributed under the terms of the Creative Commons Attribution License (http://creativecommons.org/licenses/by/2.0), which permits unrestricted use, distribution, and reproduction in any medium, provided the original work is properly cited. 
where $f$ is a contraction on $H$. They proved the above sequence $\left\{x_{n}\right\}$ converges strongly to the unique solution of the variational inequality

$$
\left\langle(A-\gamma f) x^{*}, x-x^{*}\right\rangle \geq 0, \quad \forall x \in \operatorname{Fix}(T),
$$

which is the optimality condition for the minimization problem

$$
\min _{x \in \operatorname{Fix}(T)} \frac{1}{2}\langle A x, x\rangle-h(x)
$$

where $h$ is a potential function for $\gamma f\left(\right.$ i.e., $h^{\prime}(x)=\gamma f(x)$ for $x \in H$ ).

In 2001, Yamada [3] considered the following hybrid iterative method:

$$
x_{n+1}=T x_{n}-\mu \lambda_{n} F\left(T x_{n}\right)
$$

where $F$ is $L$-Lipschitzian continuous and $\eta$-strongly monotone operator with $L>0, \eta>0$ and $0<\mu<2 \eta / L^{2}$. Under some appropriate conditions, the sequence $\left\{x_{n}\right\}$ generated by (1.4) converges strongly to the unique solution of the variational inequality

$$
\left\langle F x^{*}, x-x^{*}\right| \geq 0, \quad \forall x \in \operatorname{Fix}(T)
$$

Combining (1.3) and (1.4), Tian [4] considered the following general viscosity type iterative method:

$$
x_{n+1}=\alpha_{n} \gamma f\left(x_{n}\right)+\left(I-\mu \alpha_{n} F\right) T x_{n} .
$$

Improving and extending the corresponding results given by Marino et al., he proved that the sequence $\left\{x_{n}\right\}$ generated by (1.5) converges strongly to the unique solution $x^{*} \in \operatorname{Fix}(T)$ of the variational inequality

$$
\langle(\gamma f-\mu F) \tilde{x}, x-\tilde{x}\rangle \leq 0, \quad \forall x \in \operatorname{Fix}(T)
$$

In [5], Tian generalized the iterative method (1.5) replacing the contraction operator $f$ with a Lipschitzian continuous operator $V$ to solve the following variational inequality:

$$
\langle(\gamma V-\mu F) \tilde{x}, x-\tilde{x}\rangle \leq 0, \quad \forall x \in \operatorname{Fix}(T) .
$$

On the other hand, let $\left\{T_{i}\right\}_{i=1}^{N}$ be a finite family of nonexpansive self-mappings of $H$. Assume $\bigcap_{i=1}^{N} \operatorname{Fix}\left(T_{i}\right) \neq \emptyset$. In [1], Xu also defined the following sequence $\left\{x_{n}\right\}$ :

$$
x_{n+1}=\alpha_{n} u+\left(I-\alpha_{n} A\right) T_{n+1} x_{n}, \quad n \geq 0,
$$

where $T_{n}=T_{n \bmod N}$ and the mod function takes values in $\{1,2, \ldots, N\}$. He found that the sequence $\left\{x_{n}\right\}$ generated by (1.7) converges strongly to the unique solution of the minimization problem (1.1) with $C=\bigcap_{i=1}^{N} \operatorname{Fix}\left(T_{i}\right)$ under suitable conditions on $\left\{\alpha_{n}\right\}$ and the following additional condition on $\left\{T_{n}\right\}$ :

$$
F\left(T_{N} \cdots T_{2} T_{1}\right)=F\left(T_{1} T_{N} \cdots T_{3} T_{2}\right)=\cdots=F\left(T_{N-1} \cdots T_{1} T_{N}\right) .
$$

In fact, there are many nonexpansive mappings which do not satisfy (1.8). 
In 1999, Atsushiba and Takahashi [6] defined the $W_{n}$-mappings generated by $T_{1}, T_{2}, \ldots$, $T_{N}$ and $\left\{\gamma_{n, 1}\right\},\left\{\gamma_{n, 2}\right\}, \ldots,\left\{\gamma_{n, N}\right\} \subset[0,1]$ as follows:

$$
\begin{aligned}
& U_{n, 0}=I, \\
& U_{n, 1}=\gamma_{n, 1} T_{1} U_{n, 0}+\left(1-\gamma_{n, 1}\right) I, \\
& U_{n, 2}=\gamma_{n, 2} T_{2} U_{n, 1}+\left(1-\gamma_{n, 2}\right) I, \\
& \vdots \\
& U_{n, N-1}=\gamma_{n, N-1} T_{N-1} U_{n, N-2}+\left(1-\gamma_{n, N-1}\right) I, \\
& W_{n}=U_{n, N}=\gamma_{n, N} T_{N} U_{n, N-1}+\left(1-\gamma_{n, N}\right) I .
\end{aligned}
$$

From [6, Lemma 3.1], we know that $F\left(W_{n}\right)=\bigcap_{i=1}^{N} F\left(T_{i}\right)$.

In 2006, Yao [7] introduced the following iterative method:

$$
x_{n+1}=\alpha_{n} \gamma f\left(x_{n}\right)+\beta x_{n}+\left((1-\beta) I-\alpha_{n} A\right) W_{n} x_{n} .
$$

Without the condition (1.8), he proved that the sequence $\left\{x_{n}\right\}$ generated by (1.9) converges strongly to the unique solution of the following variational inequality:

$$
\left\langle(A-\gamma f) x^{*}, x^{*}-x\right\rangle \leq 0, \quad \forall x \in \bigcap_{i=1}^{N} \operatorname{Fix}\left(T_{i}\right),
$$

which is the optimality condition for the minimization problem

$$
\min _{x \in C} \frac{1}{2}\langle A x, x\rangle-h(x)
$$

where $C=\bigcap_{i=1}^{N} \operatorname{Fix}\left(T_{i}\right)$ and $h$ is a potential function for $\gamma f\left(i . e ., h^{\prime}(x)=\gamma f(x)\right)$.

Shang et al. [8] introduced the following scheme:

$$
\left\{\begin{array}{l}
y_{n}=\beta_{n} x_{n}+\left(1-\beta_{n}\right) W_{n} x_{n}, \\
x_{n+1}=\alpha_{n} \gamma f\left(x_{n}\right)+\left(I-\alpha_{n} A\right) y_{n} .
\end{array}\right.
$$

Under certain appropriate conditions, without (1.8), they proved that $\left\{x_{n}\right\}$ defined by (1.12) converges strongly to the unique solution of $(1.10)$ which is also the optimality condition for (1.11).

Recently, combining the Krasnoselskii-Mann type algorithm and the steepest-descent method, Buong and Duong [9] introduced a new explicit iterative algorithm:

$$
x_{k+1}=\left(1-\beta_{k}^{0}\right) x_{k}+\beta_{k}^{0} T_{0}^{k} T_{N}^{k} \cdots T_{1}^{k} x_{k}
$$

where $T_{i}^{k}=\left(1-\beta_{k}^{i}\right) I+\beta_{k}^{i} T_{i}$ for $i=1,2, \ldots, N, T_{0}^{k}=I-\lambda_{k} \mu F$, and $F$ is an $L$-Lipschitz continuous and $\eta$-strongly monotone mapping. Under some appropriate assumptions, they proved that the sequence $\left\{x_{k}\right\}$ converges strongly to the unique solution of the following 
variational inequality:

$$
\left\langle F\left(x^{*}\right), x-x^{*}\right\rangle \geq 0, \quad \forall x \in \bigcap_{i=1}^{N} \operatorname{Fix}\left(T_{i}\right) .
$$

Very recently, Zhou and Wang [10] proposed a simpler iterative algorithm than the iterative algorithm (1.13) given by Buong and Duong:

$$
x_{k+1}=\left(I-\lambda_{k} \mu F\right) T_{N}^{k} \cdots T_{1}^{k} x_{k} .
$$

They proved that the sequence $\left\{x_{k}\right\}$ defined by (1.15) converges strongly to the unique solution of the variational inequality (1.14) in a faster rate of convergence.

Motivated and inspired by the results of Zhou et al., in this paper, we consider a new iterative algorithm to solve the class of variational inequalities (1.6). The iterative algorithm improves and extends the results of Yao et al., and the corresponding results announced by many others. At the end of this paper, we extend our iterative algorithm to the more broad family of $\lambda$-strictly pseudo-contractive mappings.

\section{Preliminaries}

Throughout this paper, we write $x_{n} \rightarrow x$ and $x_{n} \rightarrow x$ to indicate that $\left\{x_{n}\right\}$ converges weakly to $x$ and converges strongly to $x$, respectively.

An operator $T: H \rightarrow H$ is said to be nonexpansive if $\|T x-T y\| \leq\|x-y\|$ for all $x, y \in H$. It is well known that $\operatorname{Fix}(T)$ is closed and convex. $A$ is called strongly positive if there exists a constant $\gamma>0$ such that $\langle A x, x\rangle \geq \gamma\|x\|^{2}$ for all $x \in H$. The operator $F$ is called $\eta$-strongly monotone if there exists a constant $\eta>0$ such that

$$
\langle x-y, F x-F y\rangle \geq \eta\|x-y\|^{2}
$$

for all $x, y \in H$.

In order to prove our results, we collect some necessary conceptions and lemmas in this section.

Definition 2.1 A mapping $T: H \rightarrow H$ is said to be an averaged mapping if there exists some number $\alpha \in(0,1)$ such that

$$
T=(1-\alpha) I+\alpha S
$$

where $I: H \rightarrow H$ is the identity mapping and $S: H \rightarrow H$ is nonexpansive. More precisely, when (2.1) holds, we say that $T$ is $\alpha$-averaged.

Lemma 2.1 ([11]) (i) The composite of finitely many averaged mappings is averaged. That is, if each of the mappings $\left\{T_{i}\right\}_{i=1}^{N}$ is averaged, then so is the composite $T_{1} \cdots T_{N}$. In particular, if $T_{1}$ is $\alpha_{1}$-averaged and $T_{2}$ is $\alpha_{2}$-averaged, where $\alpha_{1}, \alpha_{2} \in(0,1)$, then both $T_{1} T_{2}$ and $T_{2} T_{1}$ are $\alpha$-averaged, where $\alpha=\alpha_{1}+\alpha_{2}-\alpha_{1} \alpha_{2}$.

(ii) If the mappings $\left\{T_{i}\right\}_{i=1}^{N}$ are averaged and have a common fixed point, then

$$
\bigcap_{i=1}^{N} \operatorname{Fix}\left(T_{i}\right)=\operatorname{Fix}\left(T_{1} \cdots T_{N}\right) .
$$

In particular, if $N=2$, we have $\operatorname{Fix}\left(T_{1}\right) \cap \operatorname{Fix}\left(T_{2}\right)=\operatorname{Fix}\left(T_{1} T_{2}\right)=\operatorname{Fix}\left(T_{2} T_{1}\right)$. 
Lemma 2.2 ([12]) Let $C$ be a closed convex subset of a real Hilbert space $H$. Given $x \in H$ and $y \in C$. Then $y=P_{C} x$ if and only if the following inequality holds:

$$
\langle x-y, z-y\rangle \leq 0
$$

for every $z \in C$.

Lemma 2.3 ([5]) Assume $V$ is a contraction on a Hilbert space $H$ with coefficient $\alpha>0$, and $F: H \rightarrow H$ is an L-Lipschitzian continuous and $\eta$-strongly monotone operator with $L>0, \eta>0$. Then, for $0<\gamma<\frac{\mu \eta}{\alpha}, \mu F-\gamma V$ is strongly monotone with coefficient $\mu \eta-\gamma \alpha$.

Lemma 2.4 ([13]) Let $H$ be a Hilbert space, $C$ a closed convex subset of $H$, and $T: C \rightarrow C$ a nonexpansive mapping with $\operatorname{Fix}(T) \neq \emptyset$. If $\left\{x_{n}\right\}$ is a sequence in $C$ weakly converging to $x \in C$ and $\left\{(I-T) x_{n}\right\}$ converges strongly to $y \in C$, then $(I-T) x=y$. In particular, if $y=0$, then $x \in \operatorname{Fix}(T)$.

Lemma 2.5 ([14]) Let $\left\{x_{n}\right\}$ and $\left\{z_{n}\right\}$ be bounded sequences in a Banach space $X$ and $\left\{\beta_{n}\right\}$ be a sequence in $[0,1]$ which satisfies the following condition:

$$
0<\liminf _{n \rightarrow \infty} \beta_{n} \leq \limsup _{n \rightarrow \infty} \beta_{n}<1 .
$$

Suppose $x_{n+1}=\left(1-\beta_{n}\right) z_{n}+\beta_{n} x_{n}$ for all integers $n \geq 0$ and

$$
\limsup _{n \rightarrow \infty}\left(\left\|z_{n+1}-z_{n}\right\|-\left\|x_{n+1}-x_{n}\right\|\right) \leq 0 .
$$

Then $\lim _{n \rightarrow \infty}\left\|z_{n}-x_{n}\right\|=0$.

Lemma 2.6 ([1]) Assume $\left\{a_{n}\right\}$ is a sequence of nonnegative real numbers such that

$$
a_{n+1} \leq\left(1-\gamma_{n}\right) a_{n}+\delta_{n}, \quad n \geq 0,
$$

where $\left\{\gamma_{n}\right\}$ is a sequence in $(0,1)$ and $\left\{\delta_{n}\right\}$ is a sequence such that

(i) $\sum_{n=1}^{\infty} \gamma_{n}=\infty$,

(ii) $\lim \sup _{n \rightarrow \infty} \frac{\delta_{n}}{\gamma_{n}} \leq 0$ or $\sum_{n=1}^{\infty}\left|\delta_{n}\right|<\infty$.

Then $\lim _{n \rightarrow \infty} a_{n}=0$.

Lemma 2.7 ([15]) Assume $S$ is a $\lambda$-strictly pseudo-contractive mapping on a Hilbert space $H$. Define a mapping $T$ by $T x=\alpha x+(1-\alpha) S x$ for all $x \in H$ and $\alpha \in[\lambda, 1)$. Then $T$ is a nonexpansive mapping such that $\operatorname{Fix}(T)=\operatorname{Fix}(S)$.

\section{Main results}

Now we state and prove our main results in this paper.

Theorem 3.1 Let $\left\{T_{i}\right\}_{i=1}^{N}$ be $N$ nonexpansive mappings of a real Hilbert space $H$ such that $C=\bigcap_{i=1}^{N} \operatorname{Fix}\left(T_{i}\right) \neq \emptyset, F$ be an L-Lipschitzian continuous and $\eta$-strongly monotone operator on $H$ with $L>0$ and $\eta>0, V$ be an $\alpha$-Lipschitzian on $H$ with $\alpha>0$. Suppose $x_{1} \in H$ and $0<\mu<\frac{2 \eta}{L^{2}}$. Define a sequence $\left\{x_{k}\right\}$ as follows:

$$
x_{k+1}=\alpha_{k} \gamma V\left(x_{k}\right)+\left(I-\mu \alpha_{k} F\right) T_{N}^{k} T_{N-1}^{k} \cdots T_{1}^{k} x_{k}, \quad k \geq 0,
$$


where $0<\gamma<\frac{\tau}{\alpha}$ with $\tau=\mu\left(\eta-\frac{1}{2} \mu L^{2}\right)$ and $T_{i}^{k}=\left(1-\beta_{k}^{i}\right) I+\beta_{k}^{i} T_{i}$ for $i=1,2, \ldots, N$. Suppose $\alpha_{k} \in(0,1)$ and $\beta_{k}^{i} \in(\xi, \zeta)$ for some $\xi, \zeta \in(0,1)$. If the following conditions are satisfied:

(i) $\lim _{k \rightarrow \infty} \alpha_{k}=0$;

(ii) $\sum_{k=1}^{\infty} \alpha_{k}=\infty$;

(iii) $\lim _{k \rightarrow \infty}\left|\beta_{k+1}^{i}-\beta_{k}^{i}\right|=0$ for $i=1,2, \ldots, N$.

Then the sequence $\left\{x_{k}\right\}$ converges strongly to the unique solution $x^{*}$ of the variational inequality:

$$
\left\langle(\mu F-\gamma V) x^{*}, x-x^{*}\right\rangle \geq 0, \quad \forall x \in \bigcap_{i=1}^{N} \operatorname{Fix}\left(T_{i}\right) .
$$

Equivalently, we have $P_{C}(I-\mu F+\gamma V) x^{*}=x^{*}$.

Proof Since our methods easily deduce the general case, we prove Theorem 3.1 for $N=2$.

First, we show $\left\{x_{k}\right\}$ is bounded. In fact, for some point $p \in C$, by (3.1) we have

$$
\begin{aligned}
\left\|x_{k+1}-p\right\| & =\left\|\alpha_{k} \gamma V x_{k}+\left(I-\mu \alpha_{k} F\right) T_{2}^{k} T_{1}^{k} x_{k}-p\right\| \\
& =\left\|\left(I-\mu \alpha_{k} F\right) T_{2}^{k} T_{1}^{k} x_{k}-\left(I-\mu \alpha_{k} F\right) p+\alpha_{k}\left(\gamma V x_{k}-\mu F p\right)\right\| \\
& \leq\left(1-\alpha_{k} \tau\right)\left\|T_{2}^{k} T_{1}^{k} x_{k}-T_{2}^{k} T_{1}^{k} p\right\|+\alpha_{k}\left(\left\|\gamma V x_{k}-\gamma V p\right\|+\|\gamma V p-\mu F p\|\right) \\
& \leq\left(1-\alpha_{k} \tau\right)\left\|x_{k}-p\right\|+\alpha_{k} \gamma \alpha\left\|x_{k}-p\right\|+\alpha_{k}\|\gamma V p-\mu F p\| \\
& =\left(1-\alpha_{k}(\tau-\gamma \alpha)\right)\left\|x_{k}-p\right\|+\alpha_{k}(\tau-\gamma \alpha) \frac{\|\gamma V p-\mu F p\|}{\tau-\gamma \alpha} \\
& \leq \max \left\{\left\|x_{k}-p\right\|, \frac{1}{\tau-\gamma \alpha}\|\gamma V p-\mu F p\|\right\} \\
& \leq \cdots \leq \max \left\{\left\|x_{0}-p\right\|, \frac{1}{\tau-\gamma \alpha}\|\gamma V p-\mu F p\|\right\} .
\end{aligned}
$$

Therefore, $\left\{x_{k}\right\}$ is bounded. Hence we also see that $\left\{T_{2}^{k} T_{1}^{k} x_{k}\right\},\left\{F T_{2}^{k} T_{1}^{k} x_{k}\right\}$, and $\left\{V x_{k}\right\}$ are all bounded. From (3.1), it follows that

$$
\lim _{k \rightarrow \infty}\left\|x_{k+1}-T_{2}^{k} T_{1}^{k} x_{k}\right\|=0
$$

We next show that $\lim _{k \rightarrow \infty}\left\|x_{k+1}-x_{k}\right\|=0$. Noting that $T_{1}^{k}$ and $T_{2}^{k}$ are $\beta_{k}^{1}$-averaged and $\beta_{k}^{2}$-averaged, respectively, by Lemma 2.1, we find that $T_{2}^{k} T_{1}^{k}$ is $t_{k}$-averaged for every $k$, where $t_{k}=\beta_{k}^{1}+\beta_{k}^{2}-\beta_{k}^{1} \beta_{k}^{2}$. Set $\xi^{*}=2 \xi-\xi^{2}$ and $\zeta^{*}=2 \zeta-\zeta^{2}$. It is easy to deduce that $0<\xi^{*} \leq t_{k} \leq \zeta^{*}<1$ for all $k$ and

$$
\lim _{k \rightarrow \infty}\left\|t_{k+1}-t_{k}\right\|=0
$$

Since for every $k, T_{2}^{k} T_{1}^{k}$ is $t_{k}$-averaged, we can find a family of nonexpansive mappings $\left\{S_{k}\right\}_{k \geq 0}$ on $H$ such that

$$
T_{2}^{k} T_{1}^{k}=\left(1-t_{k}\right) I+t_{k} S_{k}, \quad k \geq 0
$$


Substituting (3.4) into (3.1) yields

$$
\begin{aligned}
x_{k+1} & =\alpha_{k} \gamma V x_{k}+\left(I-\mu \alpha_{k} F\right)\left[\left(1-t_{k}\right) x_{k}+t_{k} S_{k} x_{k}\right] \\
& =\left(1-t_{k}\right) x_{k}+t_{k}\left[S_{k} x_{k}+\frac{\alpha_{k}}{t_{k}}\left(\gamma V x_{k}-\mu F T_{2}^{k} T_{1}^{k} x_{k}\right)\right] .
\end{aligned}
$$

Define a sequence $\left\{z_{k}\right\}$ by $z_{k}=S_{k} x_{k}+\frac{\alpha_{k}}{t_{k}}\left(\gamma V x_{k}-\mu F T_{2}^{k} T_{1}^{k} x_{k}\right)$, so

$$
x_{k+1}=\left(1-t_{k}\right) x_{k}+t_{k} z_{k}
$$

Now, we claim that

$$
\limsup _{k \rightarrow \infty}\left(\left\|z_{k+1}-z_{k}\right\|-\left\|x_{k+1}-x_{k}\right\|\right) \leq 0
$$

To this end, we observe that

$$
\begin{aligned}
\left\|z_{k+1}-z_{k}\right\| \leq & \left\|S_{k+1} x_{k+1}-S_{k} x_{k}\right\|+\frac{\alpha_{k+1}}{t_{k+1}}\left\|\gamma V x_{k+1}-\mu F T_{2}^{k+1} T_{1}^{k+1} x_{k+1}\right\| \\
& +\frac{\alpha_{k}}{t_{k}}\left\|\gamma V x_{k}-\mu F T_{2}^{k} T_{1}^{k} x_{k}\right\| \\
\leq & \left\|S_{k+1} x_{k+1}-S_{k+1} x_{k}\right\|+\left\|S_{k+1} x_{k}-S_{k} x_{k}\right\| \\
& +\frac{\alpha_{k+1}}{t_{k+1}}\left(\left\|\gamma V x_{k+1}\right\|+\left\|\mu F T_{2}^{k+1} T_{1}^{k+1} x_{k+1}\right\|\right) \\
& +\frac{\alpha_{k}}{t_{k}}\left(\left\|\gamma V x_{k}\right\|+\left\|\mu F T_{2}^{k} T_{1}^{k} x_{k}\right\|\right) \\
\leq & \left\|x_{k+1}-x_{k}\right\|+\left\|S_{k+1} x_{k}-S_{k} x_{k}\right\| \\
& +\frac{\alpha_{k+1}}{t_{k+1}}\left(\left\|\gamma V x_{k+1}\right\|+\left\|\mu F T_{2}^{k+1} T_{1}^{k+1} x_{k+1}\right\|\right) \\
& +\frac{\alpha_{k}}{t_{k}}\left(\left\|\gamma V x_{k}\right\|+\left\|\mu F T_{2}^{k} T_{1}^{k} x_{k}\right\|\right)
\end{aligned}
$$

and

$$
\begin{aligned}
\left\|S_{k+1} x_{k}-S_{k} x_{k}\right\|= & \left\|\frac{1}{t_{k+1}} T_{2}^{k+1} T_{1}^{k+1} x_{k}-\frac{1}{t_{k}} T_{2}^{k} T_{1}^{k} x_{k}-\frac{1-t_{k+1}}{t_{k+1}} x_{k}+\frac{1-t_{k}}{t_{k}} x_{k}\right\| \\
\leq & \left|\frac{t_{k+1}-t_{k}}{t_{k+1} t_{k}}\right|\left(\left\|T_{2}^{k+1} T_{1}^{k+1} x_{k}\right\|+\left\|x_{k}\right\|\right)+\frac{1}{t_{k}}\left\|T_{2}^{k+1} T_{1}^{k+1} x_{k}-T_{2}^{k} T_{1}^{k} x_{k}\right\| \\
\leq & \left|\frac{t_{k+1}-t_{k}}{t_{k+1} t_{k}}\right| M+\frac{1}{t_{k}}\left(\left\|T_{2}^{k+1} T_{1}^{k+1} x_{k}-T_{2}^{k+1} T_{1}^{k} x_{k}\right\|\right. \\
& \left.+\left\|T_{2}^{k+1} T_{1}^{k} x_{k}-T_{2}^{k} T_{1}^{k} x_{k}\right\|\right) \\
\leq & \left|\frac{t_{k+1}-t_{k}}{t_{k+1} t_{k}}\right| M+\frac{1}{\xi^{*}}\left(\left\|T_{1}^{k+1} x_{k}-T_{1}^{k} x_{k}\right\|\right. \\
& \left.+\left\|T_{2}^{k+1} T_{1}^{k} x_{k}-T_{2}^{k} T_{1}^{k} x_{k}\right\|\right),
\end{aligned}
$$

where $M$ is a fixed constant satisfying

$$
M \geq \sup _{k \geq 0}\left\{\left\|T_{2}^{k+1} T_{1}^{k+1} x_{k}\right\|+\left\|x_{k}\right\|\right\} .
$$


Note that

$$
\begin{aligned}
\left\|T_{1}^{k+1} x_{k}-T_{1}^{k} x_{k}\right\| & =\left\|\left(1-\beta_{k+1}^{1}\right) x_{k}+\beta_{k+1}^{1} T_{1} x_{k}-\left(1-\beta_{k}^{1}\right) x_{k}-\beta_{k}^{1} T_{1} x_{k}\right\| \\
& \leq\left|\beta_{k+1}^{1}-\beta_{k}^{1}\right|\left(\left\|x_{k}\right\|+\left\|T_{1} x_{k}\right\|\right) .
\end{aligned}
$$

Since $\lim _{k \rightarrow \infty}\left|\beta_{k+1}^{i}-\beta_{k}^{i}\right|=0$ for $i=1,2$, and $\left\{x_{k}\right\}$ and $\left\{T_{1} x_{k}\right\}$ are bounded, we easily obtain

$$
\lim _{k \rightarrow \infty}\left\|T_{1}^{k+1} x_{k}-T_{1}^{k} x_{k}\right\|=0
$$

Similarly,

$$
\left\|T_{2}^{k+1} T_{1}^{k} x_{k}-T_{2}^{k} T_{1}^{k} x_{k}\right\| \leq\left|\beta_{k+1}^{2}-\beta_{k}^{2}\right|\left(\left\|T_{1}^{k} x_{k}\right\|+\left\|T_{2} T_{1}^{k} x_{k}\right\|\right),
$$

from which it follows that

$$
\lim _{k \rightarrow \infty}\left\|T_{2}^{k+1} T_{1}^{k} x_{k}-T_{2}^{k} T_{1}^{k} x_{k}\right\|=0
$$

Using (3.4), (3.9), and (3.10), from (3.8) we have

$$
\lim _{k \rightarrow \infty}\left\|S_{k+1} x_{k}-S_{k} x_{k}\right\|=0 .
$$

Since $\lim _{k \rightarrow \infty} \alpha_{k}=0$ and $0<\xi^{*}<t_{k}<\zeta^{*}<1$, combining (3.7) and (3.11) we get

$$
\limsup _{k \rightarrow \infty}\left(\left\|z_{k+1}-z_{k}\right\|-\left\|x_{k+1}-x_{k}\right\|\right) \leq 0 .
$$

By Lemma 2.5, we conclude that $\lim _{k \rightarrow \infty}\left\|z_{k}-x_{k}\right\|=0$, which implies that $\lim _{k \rightarrow \infty} \| x_{k+1}-$ $x_{k} \|=0$ by (3.6). Thus from (3.3), it is true that

$$
\lim _{k \rightarrow \infty}\left\|x_{k}-T_{2}^{k} T_{1}^{k} x_{k}\right\|=0
$$

From [8, Theorem 3.2], we know that the solution of the variational inequality (3.2) is unique. We use $x^{*}$ to denote the unique solution of (3.2). Since $\left\{x_{k}\right\}_{k \geq 0}$ is bounded, there exists a subsequence $\left\{x_{k_{j}}\right\}_{j \geq 1}$ of $\left\{x_{k}\right\}_{k \geq 0}$ such that $x_{k_{j}} \rightarrow \hat{x}$ as $j \rightarrow \infty$ and

$$
\limsup _{k \rightarrow \infty}\left\langle(\mu F-\gamma V) x^{*}, x^{*}-x_{k}\right\rangle=\lim _{j \rightarrow \infty}\left\langle(\mu F-\gamma V) x^{*}, x^{*}-x_{k_{j}}\right\rangle .
$$

Since $\left\{\beta_{k}^{i}\right\}$ is bounded for $i=1,2$, we can assume that $\beta_{k_{j}}^{i} \rightarrow \beta_{\infty}^{i}$ as $j \rightarrow \infty$, where $0<\xi \leq$ $\beta_{\infty}^{i} \leq \zeta<1$ for $i=1,2$. Define $T_{i}^{\infty}=\left(1-\beta_{\infty}^{i}\right) I+\beta_{\infty}^{i} T_{i}(i=1,2)$. Then we have $\operatorname{Fix}\left(T_{i}^{\infty}\right)=$ $\operatorname{Fix}\left(T_{i}\right)$ for $i=1,2$. Note that

$$
\left\|T_{i}^{k_{j}} x-T_{i}^{\infty} x\right\| \leq\left|\beta_{k_{j}}^{i}-\beta_{\infty}^{i}\right|\left(\|x\|+\left\|T_{i} x\right\|\right) .
$$

Hence, we deduce that

$$
\lim _{j \rightarrow \infty} \sup _{x \in D}\left\|T_{i}^{k_{j}} x-T_{i}^{\infty} x\right\|=0
$$

where $D$ is an arbitrary bounded subset of $H$. 
Since $\operatorname{Fix}\left(T_{1}^{\infty}\right) \cap \operatorname{Fix}\left(T_{2}^{\infty}\right)=\operatorname{Fix}\left(T_{1}\right) \cap \operatorname{Fix}\left(T_{2}\right)=C \neq \emptyset$ and $T_{i}^{\infty}$ is $\beta_{\infty}^{i}$-averaged for $i=1,2$, by Lemma 2.1, we know that $\operatorname{Fix}\left(T_{2}^{\infty} T_{1}^{\infty}\right)=\operatorname{Fix}\left(T_{2}^{\infty}\right) \cap \operatorname{Fix}\left(T_{1}^{\infty}\right)=C$. Combining (3.12) and (3.13), we obtain

$$
\begin{aligned}
\left\|x_{k_{j}}-T_{2}^{\infty} T_{1}^{\infty} x_{k_{j}}\right\| \leq & \left\|x_{k_{j}}-T_{2}^{k_{j}} T_{1}^{k_{j}} x_{k_{j}}\right\|+\left\|T_{2}^{k_{j}} T_{1}^{k_{j}} x_{k_{j}}-T_{2}^{\infty} T_{1}^{k_{j}} x_{k_{j}}\right\| \\
& +\left\|T_{2}^{\infty} T_{1}^{k_{j}} x_{k_{j}}-T_{2}^{\infty} T_{1}^{\infty} x_{k_{j}}\right\| \\
\leq & \left\|x_{k_{j}}-T_{2}^{k_{j}} T_{1}^{k_{j}} x_{k_{j}}\right\|+\left\|T_{2}^{k_{j}} T_{1}^{k_{j}} x_{k_{j}}-T_{2}^{\infty} T_{1}^{k_{j}} x_{k_{j}}\right\| \\
& \quad+\left\|T_{1}^{k_{j}} x_{k_{j}}-T_{1}^{\infty} x_{k_{j}}\right\| \\
\leq & \left\|x_{k_{j}}-T_{2}^{k_{j}} T_{1}^{k_{j}} x_{k_{j}}\right\|+\sup _{x \in D^{\prime}}\left\|T_{2}^{k_{j}} x-T_{2}^{\infty} x\right\| \\
& +\sup _{x \in D^{\prime \prime}}\left\|T_{1}^{k_{j}} x-T_{1}^{\infty} x\right\|,
\end{aligned}
$$

where $D^{\prime}$ is a bounded subset including $\left\{T_{1}^{k_{j}} x_{k_{j}}\right\}$ and $D^{\prime \prime}$ is a bounded subset including $\left\{x_{k_{j}}\right\}$. Hence $\lim _{j \rightarrow \infty}\left\|x_{k_{j}}-T_{2}^{\infty} T_{1}^{\infty} x_{k_{j}}\right\|=0$. From Lemma 2.4, we have $\hat{x} \in \operatorname{Fix}\left(T_{2}^{\infty} T_{1}^{\infty}\right)=C$. It follows that

$$
\begin{aligned}
\limsup _{k \rightarrow \infty}\left\langle(\mu F-\gamma V) x^{*}, x^{*}-T_{2}^{k} T_{1}^{k} x_{k}\right\rangle & =\limsup _{k \rightarrow \infty}\left\langle(\mu F-\gamma V) x^{*}, x^{*}-x_{k}\right\rangle \\
& =\lim _{j \rightarrow \infty}\left\langle(\mu F-\gamma V) x^{*}, x^{*}-x_{k_{j}}\right\rangle \\
& =\left\langle(\mu F-\gamma V) x^{*}, x^{*}-\hat{x}\right\rangle \leq 0 .
\end{aligned}
$$

Finally, we show that $x_{k} \rightarrow x^{*}$ as $k \rightarrow \infty$. From (3.1), we have

$$
\begin{aligned}
\left\|x_{k+1}-x^{*}\right\|^{2}= & \left\|\alpha_{k} \gamma V x_{k}+\left(I-\mu \alpha_{k} F\right) T_{2}^{k} T_{1}^{k} x_{k}-x^{*}\right\|^{2} \\
= & \left\|\left(I-\mu \alpha_{k} F\right) T_{2}^{k} T_{1}^{k} x_{k}-\left(I-\mu \alpha_{k} F\right) x^{*}+\alpha_{k}\left(\gamma V x_{k}-\mu F x^{*}\right)\right\|^{2} \\
= & \left\|\left(I-\mu \alpha_{k} F\right) T_{2}^{k} T_{1}^{k} x_{k}-\left(I-\mu \alpha_{k} F\right) x^{*}\right\|^{2}+\alpha_{k}^{2}\left\|\gamma V x_{k}-\mu F x^{*}\right\|^{2} \\
& +2 \alpha_{k}\left(\left(I-\mu \alpha_{k} F\right) T_{2}^{k} T_{1}^{k} x_{k}-\left(I-\mu \alpha_{k} F\right) x^{*}, \gamma V x_{k}-\mu F x^{*}\right\rangle \\
\leq & \left(1-\alpha_{k} \tau\right)^{2}\left\|x_{k}-x^{*}\right\|^{2}+\alpha_{k}^{2}\left\|\gamma V x_{k}-\mu F x^{*}\right\|^{2} \\
& +2 \alpha_{k}\left(T_{2}^{k} T_{1}^{k} x_{k}-x^{*}, \gamma V x_{k}-\mu F x^{*}\right\rangle \\
& -2 \mu \alpha_{k}^{2}\left\langle F T_{2}^{k} T_{1}^{k} x_{k}-F x^{*}, \gamma V x_{k}-\mu F x^{*}\right\rangle \\
\leq & \left(1-\alpha_{k} \tau\right)^{2}\left\|x_{k}-x^{*}\right\|^{2}+\alpha_{k}^{2}\left\|\gamma V x_{k}-\mu F x^{*}\right\|^{2} \\
& +2 \alpha_{k} \gamma\left\langle T_{2}^{k} T_{1}^{k} x_{k}-x^{*}, V x_{k}-V x^{*}\right\rangle \\
& +2 \alpha_{k}\left\langle T_{2}^{k} T_{1}^{k} x_{k}-x^{*}, \gamma V x^{*}-\mu F x^{*}\right\rangle \\
& -2 \mu \alpha_{k}^{2}\left\langle F T_{2}^{k} T_{1}^{k} x_{k}-F x^{*}, \gamma V x_{k}-\mu F x^{*}\right\rangle \\
\leq & {\left[\left(1-\alpha_{k} \tau\right)^{2}+2 \alpha \alpha_{k} \gamma\right]\left\|x_{k}-x^{*}\right\|^{2}+\alpha_{k}\left[2\left\langle T_{2}^{k} T_{1}^{k} x_{k}-x^{*},(\gamma V-\mu F) x^{*}\right\rangle\right.} \\
& \left.+\alpha_{k}\left\|\gamma V x_{k}-\mu F x^{*}\right\|^{2}+2 \alpha_{k} L\left\|T_{2}^{k} T_{1}^{k} x_{k}-x^{*}\right\|\left\|\gamma V x_{k}-\mu F x^{*}\right\|\right] \\
= & {\left[1-2 \alpha_{k}(\tau-\alpha \gamma)\right]\left\|x_{k}-x^{*}\right\|^{2}+\alpha_{k}\left[2\left\langle T_{2}^{k} T_{1}^{k} x_{k}-x^{*},(\gamma V-\mu F) x^{*}\right\rangle\right.} \\
& \left.+\alpha_{k}\left(\left\|\gamma V x_{k}-\mu F x^{*}\right\|^{2}+2 L\left\|x_{k}-x^{*}\right\|\left\|\gamma V x_{k}-\mu F x^{*}\right\|+\tau^{2}\left\|x_{k}-x^{*}\right\|^{2}\right)\right]
\end{aligned}
$$




$$
\begin{aligned}
\leq & {\left[1-2 \alpha_{k}(\tau-\alpha \gamma)\right]\left\|x_{k}-x^{*}\right\|^{2} } \\
& +\alpha_{k}\left[2\left\langle T_{2}^{k} T_{1}^{k} x_{k}-x^{*},(\gamma V-\mu F) x^{*}\right\rangle+\alpha_{k} M^{\prime}\right],
\end{aligned}
$$

where $M^{\prime}$ is a constant satisfying

$$
M^{\prime} \geq \sup _{k \geq 0}\left\{\left\|\gamma V x_{k}-\mu F x^{*}\right\|^{2}+2 L\left\|T_{2}^{k} T_{1}^{k} x_{k}-x^{*}\right\|\left\|\gamma V x_{k}-\mu F x^{*}\right\|+\tau^{2}\left\|x_{k}-x^{*}\right\|^{2}\right\} .
$$

Consequently, according to the conditions (i) and (ii), (3.14), and Lemma 2.6, we conclude that $x_{k} \rightarrow x^{*}$ as $k \rightarrow \infty$. This completes the proof.

\section{An extension of our result}

In this section, we extend our result to the more broad family of $\lambda$-strictly pseudocontractive mappings. Now let us recall that a mapping $S: H \rightarrow H$ is said to be $\lambda$-strictly pseudo-contractive if there exists a constant $\lambda \in[0,1)$ such that

$$
\|S x-S y\|^{2} \leq\|x-y\|^{2}+\lambda\|(I-S) x-(I-S) y\|^{2}, \quad \forall x, y \in H .
$$

Let $\left\{S_{i}\right\}_{i=1}^{N}$ be a family of $\lambda_{i}$-strictly pseudo-contractive self-mappings of $H$ with $0 \leq$ $\lambda_{i}<1$. For $i=1,2, \ldots, N$, define

$$
\hat{T}_{i}=\omega_{i} I+\left(1-\omega_{i}\right) S_{i}
$$

where $0 \leq \lambda_{i} \leq \omega_{i}<1$. By virtue of Lemma 2.7, we know that $\left\{\hat{T}_{i}\right\}_{i=1}^{N}$ is a family of nonexpansive mappings. Thus we extend Theorem 3.1 to the family of $\lambda_{i}$-strictly pseudocontractions.

Theorem 4.1 Let $H$ be a real Hilbert space, $F: H \rightarrow H$ be an L-Lipschitizian continuous and $\eta$-strongly monotone operator on $H$ with $L>0$ and $\eta>0, V$ be an $\alpha$-Lipschitzian continuous on $H$ with $\alpha>0$. Let $\left\{S_{i}\right\}_{i=1}^{N}$ be $N \lambda_{i}$-strictly pseudo-contractive mappings on $H$ such that $C=\bigcap_{i=1}^{N} \operatorname{Fix}\left(S_{i}\right) \neq \emptyset$. Suppose $0<\mu<\frac{\tau}{\alpha}, 0<\gamma<\frac{\tau}{\alpha}$ with $\tau=\mu\left(\eta-\frac{1}{2} \mu L^{2}\right)$, $\alpha_{k} \in(0,1), \beta_{k}^{i} \in(\xi, \zeta)$ for some $\xi, \zeta \in(0,1)$ and $0 \leq \lambda_{i} \leq \omega_{i}<1$ for $i=1,2, \ldots, N$. If the conditions (i)-(iii) of Theorem 3.1 are satisfied, the sequence $\left\{x_{k}\right\}_{k \geq 0}$ defined by (3.1) with $T_{i}$ replaced by (4.1), converges strongly to the unique solution $x^{*}$ of the following variational inequality:

$$
\left\langle(\mu F-\gamma V) x^{*}, x-x^{*}\right\rangle \geq 0, \quad \forall x \in \bigcap_{i=1}^{N} \operatorname{Fix}\left(S_{i}\right)
$$

Competing interests

The authors declare that they have no competing interests.

\section{Authors' contributions}

All authors contributed equally to the writing of this paper. All authors read and approved the final manuscript.

\section{Acknowledgements}

This research is supported by the Fundamental Science Research Funds for the Central Universities (Program No. 3122013k004).

Received: 6 November 2013 Accepted: 19 February 2014 Published: 07 Mar 2014 


\section{References}

1. Xu, HK: An iterative approach to quadratic optimization. J. Optim. Theory Appl. 116, 659-678 (2003)

2. Marino, G, Xu, HK: A general iterative method for nonexpansive mappings in Hilbert spaces. J. Math. Anal. Appl. 318, 43-52 (2006)

3. Yamada, I: The hybrid steepest descent for the variational inequality problems over the intersection of fixed points sets of nonexpansive mapping. In: Butnariu, D, Censor, Y, Reich, S (eds.) Inherently Parallel Algorithms in Feasibility and Optimization and Their Application, pp. 473-504. Elsevier, New York (2001)

4. Tian, M: A general iterative algorithm for nonexpansive mappings in Hilbert spaces. Nonlinear Anal. 73, 689-694 (2010)

5. Tian, M: A general iterative method based on the hybrid steepest descent scheme for nonexpansive mappings in Hilbert spaces. In: 2010 International Conference on Computational Intelligence and Software Engineering (CiSE) (2010)

6. Atsushiba, S, Takahashi, W: Strong convergence theorems for a finite family of nonexpansive mappings and applications. Indian J. Math. 116(3), 659-678 (2003)

7. Yao, Y: A general iterative method for a finite family of nonexpansive mappings. Nonlinear Anal. 66, 2676-2687 (2007)

8. Shang, M, Su, Y, Qin, X: Strong convergence theorems for a finite family of nonexpansive mappings. Fixed Point Theory Appl. 2007, Article ID 76971 (2007). doi:10.1155/2007/76971

9. Buong, N, Duong, LT: An explicit iterative algorithm for a class of variational inequalities in Hilbert spaces. J. Optim. Theory Appl. 151, 513-524 (2011)

10. Zhou, Y, Wang, P: A simpler explicit iterative algorithm for a class of variational inequalities in Hilbert spaces. J. Optim. Theory Appl. (2013). doi:10.1007/s10957-013-0470-x (forthcoming)

11. López, G, Martin, V, Xu, HK: Iterative algorithm for the multiple-sets split feasibility problem. In: Biomedical Mathematics: Promising Directions in Imaging, Therapy Planning and Inverse Problems, pp. 243-279 (2009)

12. Marino, G, Xu, HK: Weak and strong convergence theorems for strict pseudo-contractions in Hilbert spaces. J. Math. Anal. Appl. 329, 336-346 (2007)

13. Goebel, K, Kirk, WA: Topics in Metric Fixed-Point Theory. Cambridge University Press, Cambridge (1990)

14. Suzuki, T: Strong convergence of Krasnoselskii and Mann's type sequences for one parameter nonexpansive semigroups without Bochner integrals. J. Math. Anal. Appl. 35, 227-239 (2005)

15. Zhou, Y: Convergence theorems of fixed points for $k$-strict pseudo-contractions in Hilbert spaces. Nonlinear Anal. 69 456-462 (2008)

10.1186/1687-1812-2014-60

Cite this article as: Zhang and Yang: A new explicit iterative algorithm for solving a class of variational inequalities over the common fixed points set of a finite family of nonexpansive mappings. Fixed Point Theory and Applications $2014,2014: 60$

\section{Submit your manuscript to a SpringerOpen ${ }^{\ominus}$ journal and benefit from:}

- Convenient online submission

- Rigorous peer review

- Immediate publication on acceptance

Open access: articles freely available online

- High visibility within the field

- Retaining the copyright to your article 